\title{
Sudoku Graphs are Integral
}

\author{
Torsten Sander \\ Institut für Mathematik \\ Technische Universität Clausthal \\ D-38678 Clausthal-Zellerfeld, Germany \\ e-mail: torsten.sander@math.tu-clausthal.de
}

Submitted: Mar 1, 2009; Accepted: Jul 1, 2009; Published: Jul 24, 2009

Mathematics Subject Classification: Primary 05C50, Secondary 15A18

\begin{abstract}
Sudoku graphs have only 5 or 6 distinct eigenvalues and all of them are integers. Moreover, the associated eigenspaces admit bases with entries from the set $\{0,1,-1\}$.
\end{abstract}

Keywords: Sudoku, integral graph, graph spectrum

\section{Introduction}

The recreational game of Sudoku has attained quite some popularity in recent years. A traditional Sudoku puzzle consists of a $3 \times 3$ arrangement of square blocks consisting of $3 \times 3$ cells each. Each cell may be empty or contain a number ranging from 1 to 9 , see Figure 1. The aim of the puzzle is to fill the empty cells with numbers from 1 to 9 such that every row, column and block of the puzzle contains all of the numbers $1, \ldots, 9$. A properly set up Sudoku puzzle permits only one unique way of filling the missing numbers. Many different solution techniques exist for Sudoku puzzles [6]. The game can be generalised to $n^{4}$ instead of $3^{4}=81$ cells so that numbers from 1 to $n^{2}$ need to be filled in. Let us call these puzzles $n$-Sudokus.

As a result of Sudoku's general popularity, there has also been an increasing amount of mathematical research on it. In particular, the puzzle exhibits a close connection to graph theory. Given an empty $n$-Sudoku puzzle, the corresponding Sudoku graph $\operatorname{Sud}(n)$ on $n^{4}$ vertices is derived by establishing a one-to-one mapping between the vertices and the cells and adding edges between vertices if and only if the corresponding cells are situated in the same row, column or block. This process is depicted in Figure 2.

Numbers in the cells of an $n$-Sudoku puzzle can be interpreted as a vertex colouring 


\begin{tabular}{|l|l|l|l|l|l|l|l|l|}
\hline 9 & 2 & & 1 & & 8 & 5 & & 7 \\
\hline 3 & & 1 & & & 6 & & 4 & \\
\hline 6 & 5 & & 4 & & 7 & & 3 & 2 \\
\hline 5 & 1 & 6 & 7 & & 4 & & & 8 \\
\hline & & & & 6 & 3 & & 5 & 1 \\
\hline & 9 & 3 & 5 & 8 & & 6 & 2 & \\
\hline 8 & & & & 1 & 9 & 2 & & \\
\hline 1 & & 7 & 3 & & & & & \\
\hline 2 & 3 & 9 & 8 & 7 & & 4 & 1 & 6 \\
\hline
\end{tabular}

Figure 1: Example Sudoku puzzle

of the corresponding Sudoku graph. Hence, the task of solving a Sudoku puzzle is the mathematical task of extending a partial vertex colouring to a valid $n^{2}$-colouring of the entire graph (note that the chromatic number of an $n$-Sudoku puzzle is $n^{2}$ [11]).

Mathematical research on Sudoku has mainly concentrated on aspects of colouring and isomorphism [7], [8], [9], [11], [15].

So far, it appears that no results have been published on the spectral properties of Sudoku graphs. Given a graph $G=(V, E)$ with vertex set $V=\left\{x_{1}, \ldots, x_{n}\right\}$ and edge set $E$ we define the $n \times n$ adjacency matrix $A(G)$ of $G$ (with respect to the given vertex order) with entries $a_{i j}=1$ if $x_{i} x_{j} \in E$ and $a_{i j}=0$ otherwise. Since the eigenvalues of $A(G)$ are independent of vertex order we call them the eigenvalues of the graph $G$.

We explicitly determine the eigenvalues of Sudoku graphs, which turn out to be integers. Hence, Soduku graphs belong to the important class of integral graphs. Other examples of integral graphs are the complete graphs $K_{n}[2]$, certain trees [3] and certain circulant graphs [20] (like the unitary Cayley graphs).

Moreover, we show that the associated eigenspaces admit particularly structured bases, containing only vectors with entries from the set $\{0,1,-1\}$. Such bases we call simply structured. There has been some research interest in them lately [1], [10], [13], [17], [18]. It is interesting to note that for certain molecular graphs $\{0,1,-1\}$-eigenvectors are related to equidistributivity of electron charges in non-bonding molecular orbits [19]. 


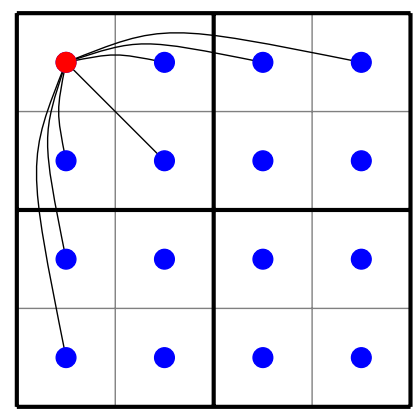

Figure 2: Deriving the graph $\operatorname{Sud}(2)$ from a 2-Sudoku puzzle

\section{NEPS}

Most of the common graph product operations can be classified as NEPS (acronym for "non-complete extended $p$-sum") operations. Given a set $B \subseteq\{0,1\}^{n} \backslash\{(0, \ldots, 0)\}$ and graphs $G_{1}, \ldots, G_{n}$, the NEPS of these graphs with respect to "basis" B is the graph $G$ with vertex set $V(G)=V\left(G_{1}\right) \times \ldots \times V\left(G_{n}\right)$ and edge set $E(G)$ such that $\left(x_{1}, \ldots, x_{n}\right),\left(y_{1} \ldots, y_{n}\right) \in V(G)$ are adjacent if and only if there exists some $n$-tuple $\left(\beta_{1}, \ldots, \beta_{n}\right) \in B$ such that $x_{i}=y_{i}$ whenever $\beta_{i}=0$ and $x_{i}, y_{i}$ are adjacent in $G_{i}$ whenever $\beta_{i}=1$.

For $n=2$, commonly used products are the direct sum $G_{1}+G_{2}$ with $B=\{(0,1),(1,0)\}$, the direct product $G_{1} \times G_{2}$ with $B=\{(1,1)\}$, and the strong product $G_{1} * G_{2}$ with $B=\{(0,1),(1,0),(1,1)\}$.

Let $A \otimes B$ denote the Kronecker product of the matrices $A, B$. According to [5], the adjacency matrix of a NEPS $G$ of graphs $G_{1}, \ldots, G_{n}$ with basis $B$ is

$$
A(G)=\sum_{\beta \in B} A\left(G_{1}\right)^{\beta_{1}} \otimes \ldots \otimes A\left(G_{n}\right)^{\beta_{n}} .
$$

We now cite a well-known result on the eigenvalues of NEPS graphs. The expression $x \otimes y$ denotes the Kronecker product of the vectors $x, y$. It is formed by replacing each entry $x_{i}$ of $x$ with the block $x_{i} y$.

Theorem 2.1. [5] For $i=1, \ldots, n$, let $\lambda_{i 1}, \ldots, \lambda_{i n_{i}}$ be the eigenvalues of the graph $G_{i}$ with $n_{i}$ vertices with respective linearly independent eigenvectors $x_{i 1}, \ldots, x_{i n_{i}}$.

Then the eigenvalues of the NEPS $G$ of $G_{1}, \ldots, G_{n}$ with basis $B$ are exactly

$$
\Lambda_{i_{1}, \ldots, i_{n}}=\sum_{\beta \in B} \lambda_{1 i_{1}}^{\beta_{1}} \cdot \ldots \cdot \lambda_{n i_{n}}^{\beta_{n}}
$$

with $i_{k}=1, \ldots, n_{k}$ for $k=1, \ldots, n$. 
With each $\Lambda_{i_{1}, \ldots, i_{n}}$ associate a vector $x_{i_{1}, \ldots, i_{n}}=x_{1 i_{1}} \otimes \ldots \otimes x_{n i_{n}}$. Then, $x_{i_{1}, \ldots, i_{n}}$ is an eigenvector of $G$ for eigenvalue $\Lambda_{i_{1}, \ldots, i_{n}}$. Together, these vectors form a complete set of linearly independent eigenvectors of $G$.

It follows from Theorem 2.1 that the eigenvalues of the direct sum $G+H$ add up (as already shown in 1947 by Rutherford [16]) whereas for the direct product they are multiplied (hence justifying the names of these operations).

Corollary 2.2. If the eigenvalues of $G_{1}$ and $G_{2}$ are integer, then also the eigenvalues of every NEPS of $G_{1}$ and $G_{2}$ are integer. If for every eigenvalue of $G_{1}$ and $G_{2}$ there exists a simply structured eigenspace basis, then this property also holds for every NEPS of $G_{1}$ and $G_{2}$.

Corollary 2.2 generalises a number of previously known results on graph products with integer eigenvalues. The special case that cube-like graphs have only integer eigenvalues dates back to 1975, cf. [12].

More information on NEPS and further generalisations can be found in [4], [5], [14].

\section{Main results}

The key to the determination of the eigenvalues of the Sudoku graphs is the observation that Sudoku graph are actually NEPS:

Lemma 3.1. Let $n \in \mathbb{N}$ and $G_{1}, \ldots, G_{4}=K_{n}$. If $G$ is the NEPS of the $G_{i}$ for basis $B=\{(0,1,0,1),(1,1,0,0),(0,0,1,1),(1,0,0,0),(0,1,0,0),(0,0,1,0),(0,0,0,1)\}$, then $G \simeq \operatorname{Sud}(n)$.

Proof. We may assume that $V\left(G_{i}\right)=\{1, \ldots, n\}$. Construct a one-to-one mapping between the 4-tuples in $V(G)=\{1, \ldots, n\}^{4}$ and the cells of the Sudoku grid as follows. For every vertex $v=(a, b, c, d) \in V(G)$, associate with $v$ the cell $\Gamma_{v}$ that lies in row number $(a-1) n+b$ and column number $(c-1) n+d$ of the Sudoku grid. Thus, $a, c$ index the vertical and horizontal block number, respectively, whereas $b, d$ index the positions inside the block. So the mapping is clearly one-to-one.

Now, fix a vertex $v \in V(G)$ and some $q \in B$ and consider how $q$ selects certain vertices of $G$ as the neighbours of $v$. We express this in terms of the associated grid cells:

- For $q=(0,1,0,1)$, select all cells in the block of $\Gamma_{v}$ that do not lie in the same row or column as $\Gamma_{v}$.

- For $q=(1,1,0,0)$, select all cells in the same column as $\Gamma_{v}$ that do not lie in the same block nor at the same relative position inside the block as $\Gamma_{v}$. 
- For $q=(1,0,0,0)$, select all cells in the same column as $\Gamma_{v}$ that do not lie in the same block but at the same relative position inside the block as $\Gamma_{v}$.

- For $q=(0,1,0,0)$, select all cells in the same column as $\Gamma_{v}$ that lie in the same block but not at the same relative position inside the block as $\Gamma_{v}$.

The remaining cases can be resolved in the same manner. Combining the cases, we find that (cf. Figure 3)

- subset $S_{1}=\{(1,1,0,0),(1,0,0,0),(0,1,0,0)\}$ selects all cells in the same column as $\Gamma_{v}$ except $\Gamma_{v}$ itself,

- subset $S_{2}=\{(0,0,1,1),(0,0,1,0),(0,0,0,1)\}$ selects all cells in the same row as $\Gamma_{v}$ except $\Gamma_{v}$ itself,

- subset $S_{3}=\{(0,1,0,1)\}$ selects all cells of the block of $\Gamma_{v}$ not selected by any of the two other subsets, with the exception of $\Gamma_{v}$ itself.

But these are exactly the adjacencies of the Sudoku graph.
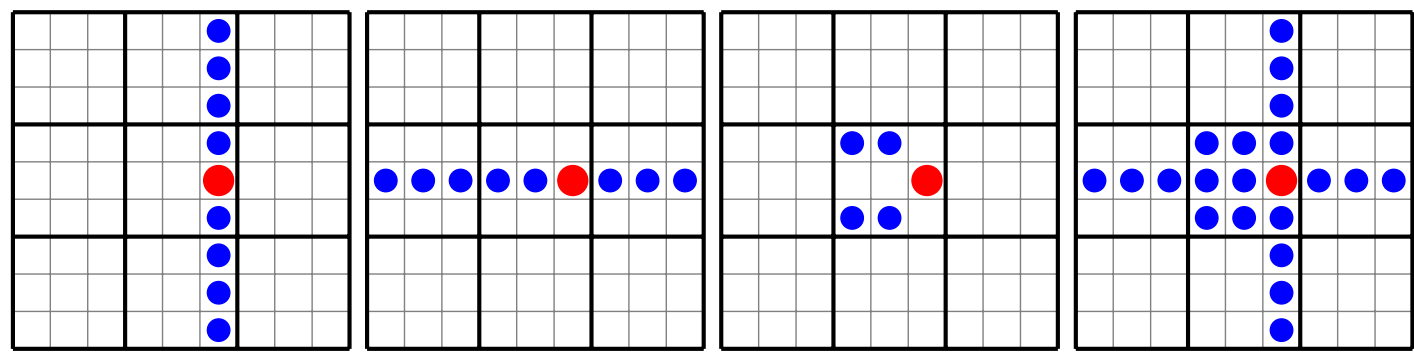

Figure 3: Selection of Sudoku cells for basis sets $S_{1}, S_{2}, S_{3}, B$

Theorem 3.2. All eigenvalues of $\operatorname{Sud}(n)$ are integers. All corresponding eigenspaces admit simply structured bases.

Proof. Observe that $K_{n}$ has single eigenvalue $n-1$ with eigenspace basis $\left\{\mathrm{j}_{n}\right\}$ and eigenvalue -1 of multiplicity $n-1$ with eigenspace basis $\left\{e_{1}-e_{2}, e_{1}-e_{3}, \ldots, e_{1}-e_{n}\right\}$. Here, $\mathrm{j}_{n}$ denotes the all ones vector of size $n$. The result now follows directly from Lemma 3.1 and Corollary 2.2.

To complete our analysis of the spectrum of Sudoku graphs, let us determine their exact eigenvalues and eigenvalue multiplicities. 
Theorem 3.3. Let $n \geq 2$. Then the spectrum of $\operatorname{Sud}(n)$, in increasing order, is

$$
\begin{aligned}
&-1-n^{\left(2 n^{3}-4 n^{2}+2 n\right)}, 1^{\left(n^{4}-2 n^{3}+n^{2}\right)}, \\
& n^{2}-2 n-1^{\left(n^{2}-2 n+1\right)}, \\
& n^{2}-n-1^{\left(2 n^{2}-2 n\right)}, \\
& 2 n^{2}-2 n-1^{(2 n-2)}, \\
& 3 n^{2}-2 n-1^{(1)} .
\end{aligned}
$$

The graph $\operatorname{Sud}(2)$ has 5 distinct eigenvalues. For $n>2$, the graph $\operatorname{Sud}(n)$ has 6 distinct eigenvalues.

Proof. According to Theorem 2.1 and Lemma 3.1, the eigenvalues of $\operatorname{Sud}(n)$ are of the form

$$
\Lambda_{\lambda_{1}, \lambda_{2}, \lambda_{3}, \lambda_{4}}=\lambda_{1} \lambda_{2}+\lambda_{2} \lambda_{4}+\lambda_{3} \lambda_{4}+\lambda_{1}+\lambda_{2}+\lambda_{3}+\lambda_{4}
$$

where the $\lambda_{i}$ are eigenvalues of $K_{n}$. Since $K_{n}$ has only eigenvalues -1 and $k=n-1$ we only need to check 16 cases so that we can conveniently determine the eigenvalues of $\operatorname{Sud}(n)$ :

$$
\begin{aligned}
-2-k & =\Lambda_{k,-1,-1, k}=\Lambda_{-1, k,-1,-1}=\Lambda_{-1,-1,-1, k}=\Lambda_{-1, k, k,-1}, \\
-1 & =\Lambda_{k,-1, k,-1}=\Lambda_{-1,-1, k,-1}=\Lambda_{-1,-1,-1,-1}=\Lambda_{k,-1,-1,-1}, \\
k^{2}-2 & =\Lambda_{-1, k,-1, k}, \\
k^{2}+k-1 & =\Lambda_{k, k,-1,-1}=\Lambda_{-1,-1, k, k}=\Lambda_{k,-1, k, k}=\Lambda_{k, k, k,-1}, \\
2 k^{2}+2 k-1 & =\Lambda_{-1, k, k, k}=\Lambda_{k, k,-1, k}, \\
3 k^{2}+4 k & =\Lambda_{k, k, k, k} .
\end{aligned}
$$

The respective multiplicities are readily concluded from this analysis and the multiplicities of the eigenvalues of $K_{n}$. It is easy to check that for $n \geq 2$ the eigenvalues can be ordered as follows:

$$
-1-n<-1 \leq n^{2}-2 n-1<n^{2}-n-1<2 n^{2}-2 n-1<3 n^{2}-2 n-1 .
$$

For $n>2$ this chain of inequalities is strict.

\section{References}

[1] Akbari, S. and Alipour, A. and Ghorbani, E. and Khosrovshahi, G. $\{-1,0,1\}$-Basis for the null space of a forest. Linear Algebra Appl., Vol. 414, pp. 506-511, 2006.

[2] Biggs, N. Algebraic graph theory. Cambridge Mathematical Library. Cambridge University Press, Cambridge, second edition, 1993. 
[3] Brouwer, A. E. Small integral trees. Electron. J. Combin. , Vol. 15, 2008.

[4] Cvetković, D., and Petrić, M. Connectedness of the non-complete extended p-sum of graphs. Zb. Rad., Prir.-Mat. Fak., Univ. Novom Sadu, Ser. Mat. , Vol. 13, pp. 345-352, 1983.

[5] Cvetković, D., Rowlinson, P., and Simić, S. Eigenspaces of graphs, Vol. 66 of Encyclopedia of Mathematics and its Applications. Cambridge University Press, Cambridge, 1997.

[6] Davis, T. The Mathematics of Sudoku. http://www.geometer.org/mathcircles/sudoku.pdf.

[7] Elsholtz, C., and Mütze, A. Sudoku im Mathematikunterricht. Math. Semesterber., Vol. 39, pp. 69-93, 2007.

[8] Felgenhauer, B., and Jarvis, F. Mathematics of Sudoku I. Math. Spectrum, Vol. 39, pp. 15-22, 2005.

[9] Harary, F., Slany, W., and Verbitsky, O. On the computational complexity of the forcing chromatic number. SIAM J. Comput., Vol. 37, pp. 1-19, 2007.

[10] Hazama, F. On the kernels of the incidence matrices of graphs. Discrete Math., Vol. 254, pp. 165-174, 2002.

[11] Herzberg, A. M., and Murty, M. R. Sudoku squares and chromatic polynomials. Notices AMS, Vol. 6, pp. 708-717, 2007.

[12] Lovász, L. Spectra of graphs with transitive groups. Periodica Math. Hung., Vol. 6, pp. 191-195, 1975.

[13] Nath, M. and Sarma, B. K. On the null-spaces of acyclic and unicyclic singular graphs. Linear Algebra Appl., Vol. 427, pp. 42-54, 2007.

[14] Petrić, M. Connectedness of the generalized direct product of digraphs. Univ. Beograd. Publ. Elektrotehn. Fak. (Ser. Mat.), Vol. 6, pp. 30-38, 1995.

[15] Russell, E., and Jarvis, F. Mathematics of Sudoku II. Math. Spectrum, Vol. 39, pp. 54-58, 2005.

[16] Rutherford, D. E. Some continuant determinants arising in physics and chemistry. Proc. Roy. Soc. Edinburgh. Sect. A., Vol. 62, pp. 229-236, 1947.

[17] Sander, J. W. and Sander, T. On Simply Structured Bases of Tree Kernels. AKCE J. Graphs. Combin., Vol. 2, No. 1, pp. 45-56, 2005.

[18] Sander, T. On certain eigenspaces of cographs. Electron. J. Combin., Vol. 15, 2008.

[19] Sciriha, I. Coalesced and Embedded Nut Graphs in Singular Graphs. Ars Math. Contemp., Vol. 1, No. 1, pp. 20-31, 2008.

[20] So, W. Integral circulant graphs. Discrete Math., Vol. 306, No. 1, pp. 153-158, 2006. 